\title{
Stochastic Programming with Cauchy Distribution
}

\author{
Manas Kumar Pal \\ Institute of Management \& Information Science \\ Bhubaneswar, Odisha, India \\ manas.sbp@gmail.com
}

\author{
S. Bagh \\ Department of Statistics, Sambalpur University \\ Orissa, India
}

\begin{abstract}
The aim of this paper is to derive a method for solving a stochastic linear programming problem with Cauchy distribution. Assuming that the coefficients are distributed as Cauchy random variables, the stochastic linear programming is converted to a deterministic non-linear programming problem by a suitable transformation. Then an algorithm can be used to solve the resulting deterministic problem .A numerical example can be considered to illustrate the above methodology.
\end{abstract}

Keywords and phrases: Stochastic programming, Cauchy random variables, Probability density functions, Levy inversion theorem.

\section{Introduction}

Charnes and Cooper [6] first introduced the stochastic programming by taking different objective functions and constraints. Various models have been suggested by several researchers and most of the probabilistic model assumes normal distribution for model coefficients.

Here we consider a stochastic programming problem with Cauchy distribution. A stochastic linear programming problem can be presented as follows:

$$
\operatorname{Max} Z=\sum_{j=1}^{n} c_{j} x_{j},
$$

Subject to the constraints,

$$
\begin{aligned}
& \operatorname{Pr} o b\left(\sum_{j=1}^{n} a_{i j} x_{j} \leq b_{i}\right) \geq 1-\alpha_{i}, i=1,2, \ldots \ldots \ldots \ldots \ldots . ., m \\
& x_{j} \geq 0, \quad j=1,2, \ldots \ldots \ldots, n,
\end{aligned}
$$

Where $0<\alpha_{\mathrm{i}}<1$ and are constants. It is assumed that $\mathrm{a}_{\mathrm{ij}}$ are independent Cauchy random variables with known distributions for $\mathrm{i}=1,2, \ldots \ldots \ldots \ldots \ldots, \mathrm{m}$ and $\mathrm{j}=1,2, \ldots \ldots \ldots \ldots, \mathrm{n}$. The main objective of this section is to find a solution for general stochastic programming problems involving Cauchy distribution in the constraints. 
First we obtain the probability density function of the linear combination of ' $n$ ' independent Cauchy random variables, then using the probability density function, the probabilistic constraints are converted to the deterministic constraints, and then the resulting non-linear deterministic problem can be solved.

Probability distribution of $U_{i}=\sum_{i=1}^{n} a_{i j} x_{j}$ and the deterministic form of the probabilistic constraint

Here we will find out the probability density function of random variable $U_{i}=\sum_{j=1}^{n} a_{i j} x_{j}$, where $a_{i j}, j=1,2 \ldots \ldots . n$ are independent Cauchy random variables and $x_{j}, j=1,2, \ldots \ldots, n$ are variables .Then using it we find a deterministic form of the probabilistic constraints in a stochastic linear programming problem. The density function of $\mathrm{U}_{\mathrm{i}}$ can be obtained by studying the special cases with $n=2$ and $n=3$. Let us consider a model involving only two random variables, the $\mathrm{i}^{\text {th }}$ probabilistic constraints can be stated by

$$
\operatorname{Pr} o b\left(a_{i 1} x_{1}+a_{i 2} x_{2}\right) \leq b_{i} \geq 1-\alpha_{i}, i=1,2, \ldots \ldots \ldots \ldots \ldots, m
$$

Where $\mathrm{a}_{\mathrm{i} 1}$ and $\mathrm{a}_{\mathrm{i} 2}$ are two independent Cauchy random variables

$$
\text { Let } \mathrm{U}_{\mathrm{i}}=\mathrm{a}_{\mathrm{i} 1} \mathrm{X}_{1}+\mathrm{a}_{\mathrm{i} 2} \mathrm{X}_{2}
$$

Then the density function of $U_{i}$ can be obtained as,

$$
\begin{aligned}
f(U) & =\frac{1}{2 \pi} \int_{-\infty}^{\infty} e^{-i t u} \varphi_{u}(t) d t \quad \text { (Using Levy inversion theorem) } \\
& =\frac{1}{2 \pi} \int_{-\infty}^{\infty} e^{-i t u} e^{-\left(x_{1}+x_{2}\right) t \mid} d t \\
& =\frac{1}{2 \pi}\left[\int_{-\infty}^{0} e^{-i t u} e^{\left(x_{1}+x_{2}\right) t} d t+\int_{0}^{\infty} e^{-i t u} e^{-\left(x_{1}+x_{2}\right) t} d t\right] \\
& =\frac{1}{2 \pi}\left[\int_{0}^{\infty} e^{i t u} e^{-\left(x_{1}+x_{2}\right) t} d t+\int_{0}^{\infty} e^{-i t u} e^{-\left(x_{1}+x_{2}\right) t} d t\right] \\
& =\frac{1}{2 \pi}\left[\int_{0}^{\infty} e^{i t u} e^{-\left(x_{1}+x_{2}\right) t} d t+\int_{0}^{\infty} e^{-i t u} e^{-\left(x_{1}+x_{2}\right) t} d t\right] \\
& =\frac{1}{2 \pi}\left[\int_{0}^{\infty} e^{-t\left(x_{1}+x_{2}-i u\right)} d t+\int_{0}^{\infty} e^{-t\left(x_{1}+x_{2}+i u\right)} d t\right] \\
& =\frac{1}{2 \pi}\left[\frac{e^{-t\left(x_{1}+x_{2}-i u\right)}}{-\left(x_{1}+x_{2}-i u\right)}+\frac{e^{-t\left(x_{1}+x_{2}+i u\right)}}{-\left(x_{1}+x_{2}+i u\right)}\right]_{0}^{\infty} \\
& =\frac{1}{2 \pi}\left[\frac{1}{\left(x_{1}+x_{2}-i u\right)}+\frac{1}{\left(x_{1}+x_{2}+i u\right)}\right]
\end{aligned}
$$




$$
\begin{aligned}
& =\frac{1}{2 \pi}\left[\frac{2\left(x_{1}+x_{2}\right)}{\left(\left(x_{1}+x_{2}\right)^{2}-i^{2} u^{2}\right)}\right] \\
& =\left[\frac{\left(x_{1}+x_{2}\right)}{\pi\left(\left(x_{1}+x_{2}\right)^{2}+u^{2}\right)}\right]
\end{aligned}
$$

Hence $\mathrm{i}^{\text {th }}$ probabilistic constraints can be made deterministic constraints by integrating the probability density function of $\mathrm{u}_{\mathrm{i}}$ as stated below,

$$
\int_{0}^{b_{i}} f\left(u_{i}\right) d u_{i} \geq 1-\alpha_{i} \quad i=1,2, \ldots \ldots \ldots \ldots \ldots, m
$$

This can be simplified as follows,

$$
\int_{0}^{b_{i}} f\left(u_{i}\right) d u_{i}=\frac{1}{\pi} \int_{0}^{b_{i}} \frac{\left(x_{1}+x_{2}\right)}{\left(\left(x_{1}+x_{2}\right)^{2}+u_{i}^{2}\right)^{2}} d u_{i}
$$

If $u_{i}=a_{i 1} x_{1}+a_{i 2} x_{2}$

Then $x_{1}=\frac{u_{i}-a_{i 2} x_{2}}{a_{i 1}}$ and $x_{2}=\frac{u_{i}-a_{i 1} x_{1}}{a_{i 2}}$

Now $\quad x_{1}+x_{2}=\frac{u_{i}-a_{i 2} x_{2}}{a_{i 1}}+\frac{u_{i}-a_{i 1} x_{1}}{a_{i 2}}$

$$
=\frac{u_{i}\left(a_{i 1}+a_{i 2}\right)-\left(a_{i 1}{ }^{2} x_{1}+a_{i 2}{ }^{2} x_{2}\right)}{a_{i 1} a_{i 2}}
$$

Let $A=\frac{a_{i 1}+a_{i 2}}{a_{i 1} a_{i 2}}$ and $B=\frac{a_{i 1}{ }^{2} x_{1}+a_{i 2}{ }^{2} x_{2}}{a_{i 1} a_{i 2}}$

Then, $x_{1}+x_{2}=A u_{i}-B=p($ Say $)$

Now putting the above values in (5)

$$
\begin{aligned}
\int_{0}^{b_{i}} f\left(u_{i}\right) d u_{i} & =\frac{1}{\pi} \int_{0}^{b_{i}} \frac{\left(A u_{i}-B\right)}{\left(\left(A u_{i}-B\right)^{2}+u_{i}^{2}\right)^{2}} d u_{i} \\
& =\frac{1}{\pi} \int_{-\mathbf{B}}^{A b_{i}-B} \frac{p}{A\left(p^{2}+\left(\frac{p+B}{A}\right)^{2}\right)} d p \\
& =\frac{1}{\pi} \int_{-\mathbf{B}}^{A b_{i}-B} \frac{p d p}{A\left(p^{2}+\left(\frac{p}{A}+\frac{B}{A}\right)^{2}\right)} \\
& =\frac{1}{\pi} \int_{-\mathbf{B}}^{A b_{i}-B} \frac{p d p}{A\left(p^{2}+\left(\frac{p}{A}\right)^{2}+\left(\frac{B}{A}\right)^{2}+2\left(\frac{p}{A}\right)\left(\frac{B}{A}\right)\right)}
\end{aligned}
$$




$$
\begin{aligned}
& =\frac{1}{\pi} \int_{-\mathbf{B}}^{A b_{i}-B} \frac{p d p}{A\left\{\left(1+\frac{1}{A^{2}}\right) p^{2}+\left(\frac{2 B}{A^{2}}\right) p+\frac{B^{2}}{A^{2}}\right\}} \\
& =\frac{1}{\pi} \int_{-\mathbf{B}}^{A b_{i}-B} \frac{p d p}{A\left\{\left(\frac{A^{2}+1}{A^{2}}\right) p^{2}+\left(\frac{2 B}{A^{2}}\right) p+\frac{B^{2}}{A^{2}}\right\}} \\
& =\frac{1}{\pi} \int_{-\mathbf{B}}^{A b_{i}-B} \frac{p d p}{\left\{\left(\frac{A^{2}+1}{A}\right) p^{2}+\left(\frac{2 B}{A}\right) p+\frac{B^{2}}{A}\right\}}
\end{aligned}
$$

Let $\mathrm{R}=\mathrm{a}+\mathrm{bt}+\mathrm{ct}^{2}$, where $c=\frac{A^{2}+1}{A}, b=\frac{2 B}{A}$ and $a=\frac{B^{2}}{A}$ then,

$$
\begin{aligned}
& \Delta=4 a c-b^{2}=4 B^{2}>0 \text { and } \int \frac{t d t}{R}=\frac{1}{2 c} \ln R-\frac{b}{2 c} \int \frac{d t}{R} \quad \text { (c.f [42], Page 68) } \\
& =\frac{1}{\pi}\left[\left\{\frac{1}{2\left(\frac{A^{2}+1}{A}\right)}\right\} \ln \left\{\left(\frac{A^{2}+1}{A}\right) p^{2}+\left(\frac{2 B}{A}\right) p+\frac{B^{2}}{A}\right\}-\frac{\left(\frac{2 B}{A}\right)}{2\left(\frac{A^{2}+1}{A}\right)} \int_{-B}^{A b_{i}-B} \frac{d p}{\left.\left.\int\left(\frac{A^{2}+1}{A}\right) p^{2}+\left(\frac{2 B}{A}\right) p+\frac{B^{2}}{A}\right\}\right]}\right. \\
& =\frac{1}{\pi}\left[\left\{\frac{1}{2\left(\frac{A^{2}+1}{A}\right)}\right\} \ln \left\{\left(\frac{A^{2}+1}{A}\right) p^{2}+\left(\frac{2 B}{A}\right) p+\frac{B^{2}}{A}\right\}-\frac{B}{A^{2}+1} \frac{2}{\sqrt{4 B^{2}}} \operatorname{arctg} \frac{\frac{2 B}{A}+2\left(\frac{A^{2}+1}{A}\right) p}{\sqrt{4 B^{2}}}\right]_{-B}^{A b_{i}-B}
\end{aligned}
$$

Because $\int \frac{d t}{R}=\frac{2}{\sqrt{\Delta}} \operatorname{arctg}\left(\frac{b+2 c t}{\sqrt{\Delta}}\right), i f \Delta>0 \quad$ (c.f [42], Page 68)

$$
\begin{aligned}
& =\frac{1}{\pi}\left[\left\{\frac{1}{2\left(\frac{A^{2}+1}{A}\right)}\right\} \ln \left\{\left(\frac{A^{2}+1}{A}\right) p^{2}+\left(\frac{2 B}{A}\right) p+\frac{B^{2}}{A}\right\}-\frac{1}{A^{2}+1} \operatorname{arctg}\left\{\frac{1}{A}+\left(\frac{A^{2}+1}{A B}\right) p\right\}\right]_{-B}^{A b_{i}-B} \\
& =\frac{1}{\pi}\left[\left\{\frac{1}{2\left(\frac{A^{2}+1}{A}\right)}\right\} \ln \left\{\left(\frac{A^{2}+1}{A}\right)\left(A b_{i}-B\right)^{2}+\left(\frac{2 B}{A}\right)\left(A b_{i}-B\right)+\frac{B^{2}}{A}\right\}-\frac{1}{A^{2}+1} \operatorname{arctg}\left\{\frac{1}{A}+\left(\frac{A^{2}+1}{A B}\right)\left(A b_{i}-B\right)\right\}\right]
\end{aligned}
$$




$$
\begin{aligned}
& -\frac{1}{\pi}\left[\left\{\frac{1}{2\left(\frac{A^{2}+1}{A}\right)}\right\} \ln \left\{\left(\frac{A^{2}+1}{A}\right)(-B)^{2}+\left(\frac{2 B}{A}\right)(-B)+\frac{B^{2}}{A}\right\}-\frac{1}{A^{2}+1} \operatorname{arctg}\left\{\frac{1}{A}+\left(\frac{A^{2}+1}{A B}\right)(-B)\right\}\right] \\
& =\frac{1}{\pi}\left[\begin{array}{r}
\left\{\frac{A}{2\left(A^{2}+1\right)}\right\} \ln \left\{\frac{\left(A^{2}+1\right)\left(A^{2} b_{i}^{2}+B^{2}-2 A b_{i} B\right)}{A}+\frac{2 A b_{i} B}{A}-\frac{2 B^{2}}{A}+\frac{B^{2}}{A}\right\} \\
\left.-\frac{1}{A^{2}+1} \operatorname{arctg}\left\{\frac{1}{A}+\frac{A^{3} b_{i}-A^{2} B+A b_{i}-B}{A B}\right\}\right]
\end{array}\right] \\
& -\frac{1}{\pi}\left[\left\{\frac{A}{2\left(A^{2}+1\right)}\right\} \ln \left\{\frac{A^{2} B^{2}}{A}+\frac{B^{2}}{A}-\frac{2 B^{2}}{A}+\frac{B^{2}}{A}\right\}-\frac{1}{A^{2}+1} \operatorname{arctg}\left\{\frac{1}{A}-\frac{A^{2} B}{A B}-\frac{B}{A B}\right\}\right] \\
& =\frac{1}{\pi}\left[\begin{array}{c}
\left\{\frac{A}{2\left(A^{2}+1\right)}\right\} \ln \left\{\frac{A^{4} b_{i}^{2}+A^{2} B^{2}-2 A^{3} b_{i} B+A^{2} b_{i}^{2}+B^{2}-2 A b_{i} B}{A}+2 b_{i} B-\frac{B^{2}}{A}\right\} \\
-\frac{1}{A^{2}+1} \operatorname{arctg}\left(\frac{1}{A}+\frac{A^{2} b_{i}}{B}+\frac{b_{i}}{B}-\frac{1}{A}-A\right)
\end{array}\right] \\
& -\frac{1}{\pi}\left[\left\{\frac{A}{2\left(A^{2}+1\right)}\right\} \ln \left(A B^{2}\right)-\frac{1}{A^{2}+1} \operatorname{arctg}(-A)\right] \\
& {\left[\frac{A}{2\left(A^{2}+1\right)}\left\{\ln \left(A^{3} b_{i}{ }^{2}+A B^{2}-2 A^{2} b_{i} B+A b_{i}{ }^{2}+\frac{B^{2}}{A}-2 b_{i} B+2 b_{i} B-\frac{B^{2}}{A}\right)-\ln \left(A B^{2}\right)\right\}\right.} \\
& =\frac{1}{\pi}\left(-\frac{1}{A^{2}+1}\left\{\operatorname{arctg}\left(\frac{A^{2} b_{i}}{B}+\frac{b_{i}}{B}-A\right)-\operatorname{arctg}(-A)\right\}\right. \\
& \frac{A}{2\left(A^{2}+1\right)} \ln \left\{\frac{\left(A^{2}+1\right) A b_{i}{ }^{2}-2 A^{2} b_{i} B+A B^{2}}{\left(A B^{2}\right)}\right\} \\
& =\frac{1}{\pi}\left\{\quad-\frac{1}{A^{2}+1}\left\{\operatorname{arctg}\left(\frac{A^{2} b_{i}}{B}+\frac{b_{i}}{B}-A\right)-\operatorname{arctg}(-A)\right\}\right. \\
& =\frac{1}{\pi}\left[\frac{A}{2\left(A^{2}+1\right)} \ln \left\{\frac{\left(A^{2}+1\right) A b_{i}^{2}}{A B^{2}}-\frac{2 A^{2} B b_{i}}{A B^{2}}+\frac{\left(A^{2}+1\right) A b_{i}^{2}}{A B^{2}}\right\}-\frac{1}{A^{2}+1}\left\{\operatorname{arctg}\left(\frac{A^{2} b_{i}}{B}+\frac{b_{i}}{B}-A\right)-\operatorname{arctg}(-A)\right\}\right]
\end{aligned}
$$




$$
=\frac{1}{\pi}\left[\frac{A}{2\left(A^{2}+1\right)} \ln \left\{\left(\frac{A^{2}+1}{B^{2}}\right) b_{i}^{2}-\frac{2 A b_{i}}{B}+1\right\}-\frac{1}{A^{2}+1}\left\{\operatorname{arctg}\left(\frac{b_{i}}{B}\left(A^{2}+1\right)-A\right)-\operatorname{arctg}(-A)\right\}\right]
$$

On substituting the values of $\mathrm{A}$ and $\mathrm{B}$,

$$
\left.\begin{array}{l}
\int_{0}^{b_{i}} f\left(u_{i}\right) d u_{i}= \\
\frac{1}{\pi}\left[\frac{\left(\frac{1}{a_{i 1}}+\frac{1}{a_{i 2}}\right)}{2\left\{\left(\frac{1}{a_{i 1}}+\frac{1}{a_{i 2}}\right)^{2}+1\right\}} \ln \left\{\frac{\left(\frac{1}{a_{i 1}}+\frac{1}{a_{i 2}}\right)^{2}+1}{\left(x_{1} \frac{a_{i 1}}{a_{i 2}}+x_{2} \frac{a_{i 2}}{a_{i 1}}\right)^{2}} b_{i}^{2}-\frac{2\left(\frac{1}{a_{i 1}}+\frac{1}{a_{i 2}}\right) b_{i}}{\left(x_{1} \frac{a_{i 1}}{a_{i 2}}+x_{2} \frac{a_{i 2}}{a_{i 1}}\right)}+1\right\}\right. \\
\left.-\frac{1}{\left(\frac{1}{a_{i 1}}+\frac{1}{a_{i 2}}\right)^{2}+1}\left\{\operatorname{arctg}\left(\frac{b_{i}\left(\left(\frac{1}{a_{i 1}}+\frac{1}{a_{i 2}}\right)^{2}+1\right)}{\left(x_{1} \frac{a_{i 1}}{a_{i 2}}+x_{2} \frac{a_{i 2}}{a_{i 1}}\right)}-\left(\frac{1}{a_{i 1}}+\frac{1}{a_{i 2}}\right)\right)-\operatorname{arctg}-\left(\frac{1}{a_{i 1}}+\frac{1}{a_{i 2}}\right)\right\}\right]
\end{array}\right]
$$

Proceeding as in above for the cases of three variables, the $\mathrm{i}^{\text {th }}$ probabilistic constraints can be presented as,

$$
\operatorname{Pr} o b\left(a_{i 1} x_{1}+a_{i 2} x_{2}+a_{i 3} x_{3}\right) \leq b_{i} \geq 1-\alpha_{i}, i=1,2, \ldots \ldots \ldots \ldots \ldots, m
$$

Where $\mathrm{a}_{\mathrm{i} 1}, \mathrm{a}_{\mathrm{i} 2}$ and $\mathrm{a}_{\mathrm{i} 3}$ are three independent Cauchy random variables

$$
\text { Let } U_{i}=a_{i 1} x_{1}+a_{i 2} x_{2}+a_{i 3} x_{3}
$$

Then the density function of $U_{i}$ can be obtained as,

$$
\begin{aligned}
f(U) & =\frac{1}{2 \pi} \int_{-\infty}^{\infty} e^{-i t u} \varphi_{u}(t) d t \quad \text { (Using Levy inversion theorem ) } \\
& =\frac{1}{2 \pi} \int_{-\infty}^{\infty} e^{-i t u} e^{-\left(x_{1}+x_{2}+x_{3}\right)|t|} d t \\
& =\frac{1}{2 \pi}\left[\int_{-\infty}^{0} e^{-i t u} e^{\left(x_{1}+x_{2}+x_{3}\right) t} d t+\int_{0}^{\infty} e^{-i t u} e^{-\left(x_{1}+x_{2}+x_{3}\right) t} d t\right] \\
& =\frac{1}{2 \pi}\left[\int_{0}^{\infty} e^{i t u} e^{-\left(x_{1}+x_{2}+x_{3}\right) t} d t+\int_{0}^{\infty} e^{-i t u} e^{-\left(x_{1}+x_{2}+x_{3}\right) t} d t\right] \\
& =\frac{1}{2 \pi}\left[\int_{0}^{\infty} e^{i t u} e^{-\left(x_{1}+x_{2}+x_{3}\right) t} d t+\int_{0}^{\infty} e^{-i t u} e^{-\left(x_{1}+x_{2}+x_{3}\right) t} d t\right] \\
& =\frac{1}{2 \pi}\left[\int_{0}^{\infty} e^{-t\left(x_{1}+x_{2}+x_{3}-i u\right)} d t+\int_{0}^{\infty} e^{-t\left(x_{1}+x_{2}+x_{3}+i u\right)} d t\right]
\end{aligned}
$$




$$
\begin{aligned}
& =\frac{1}{2 \pi}\left[\frac{e^{-t\left(x_{1}+x_{2}+x_{3}-i u\right)}}{-\left(x_{1}+x_{2}+x_{3}-i u\right)}+\frac{e^{-t\left(x_{1}+x_{2}+x_{3}+i u\right)}}{-\left(x_{1}+x_{2}+x_{3}+i u\right)}\right]_{0}^{\infty} \\
& =\frac{1}{2 \pi}\left[\frac{1}{\left(x_{1}+x_{2}+x_{3}-i u\right)}+\frac{1}{\left(x_{1}+x_{2}+x_{3}+i u\right)}\right] \\
& =\frac{1}{2 \pi}\left[\frac{2\left(x_{1}+x_{2}+x_{3}\right)}{\left(\left(x_{1}+x_{2}+x_{3}\right)^{2}-i^{2} u^{2}\right)}\right] \\
& =\left[\frac{\left(x_{1}+x_{2}+x_{3}\right)}{\pi\left(x_{1}+x_{2}+x_{3}^{2}+u^{2}\right)}\right]
\end{aligned}
$$

Hence $i^{\text {th }}$ probabilistic constraints can be made deterministic constraints by integrating the probability density function of $\mathrm{u}_{\mathrm{i}}$ as stated below,

$$
\int_{0}^{b_{i}} f\left(u_{i}\right) d u_{i} \geq 1-\alpha_{i} \quad i=1,2, \ldots \ldots \ldots \ldots \ldots, m
$$

This can be simplified as follows,

$$
\int_{0}^{b_{i}} f\left(u_{i}\right) d u_{i}=\frac{1}{\pi} \int_{0}^{b_{i}} \frac{\left(x_{1}+x_{2}+x_{3}\right)}{\left(\left(x_{1}+x_{2}+x_{3}\right)^{2}+u_{i}^{2}\right)} d u_{i}
$$

If $u_{i}=a_{i 1} x_{1}+a_{i 2} x_{2}+a_{i 3} x_{3}$

Then $x_{1}=\frac{u_{i}-a_{i 2} x_{2}-a_{i 3} x_{3}}{a_{i 1}}$,

$$
x_{2}=\frac{u_{i}-a_{i 1} x_{1}-a_{i 3} x_{3}}{a_{i 2}}
$$

and $\quad x_{3}=\frac{u_{i}-a_{i 1} x_{1}-a_{i 2} x_{2}}{a_{i 3}}$

Now $x_{1}+x_{2}+x_{3}=\frac{u_{i}-a_{i 2} x_{2}-a_{i 3} x_{3}}{a_{i 1}}+\frac{u_{i}-a_{i 1} x_{1}-a_{i 3} x_{3}}{a_{i 2}}+\frac{u_{i}-a_{i 1} x_{1}-a_{i 2} x_{2}}{a_{i 3}}$

$$
\begin{gathered}
=u_{i}\left(\frac{1}{a_{i 1}}+\frac{1}{a_{i 2}}+\frac{1}{a_{i 3}}\right)-\left\{x_{1}\left(\frac{a_{i 1}}{a_{i 2}}+\frac{a_{i 1}}{a_{i 3}}\right)+x_{2}\left(\frac{a_{i 2}}{a_{i 3}}+\frac{a_{i 2}}{a_{i 1}}\right)+x_{1}\left(\frac{a_{i 3}}{a_{i 1}}+\frac{a_{i 3}}{a_{i 2}}\right)\right\} \\
A=\frac{a_{i 1}+a_{i 2}+a_{i 3}}{a_{i 1} a_{i 2} a_{i 3}}
\end{gathered}
$$

Let

$$
\text { and } B=\left\{x_{1}\left(\frac{a_{i 1}}{a_{i 2}}+\frac{a_{i 1}}{a_{i 3}}\right)+x_{2}\left(\frac{a_{i 2}}{a_{i 3}}+\frac{a_{i 2}}{a_{i 1}}\right)+x_{1}\left(\frac{a_{i 3}}{a_{i 1}}+\frac{a_{i 3}}{a_{i 2}}\right)\right\}
$$

Then, $x_{1}+x_{2}+x_{3}=A u_{i}-B=p($ Say $)$

Now putting the above values in (8)

$$
\int_{0}^{b_{i}} f\left(u_{i}\right) d u_{i}=\frac{1}{\pi} \int_{0}^{b_{i}} \frac{\left(A u_{i}-B\right)}{\left(\left(A u_{i}-B\right)^{2}+u_{i}^{2}\right)} d u_{i}
$$




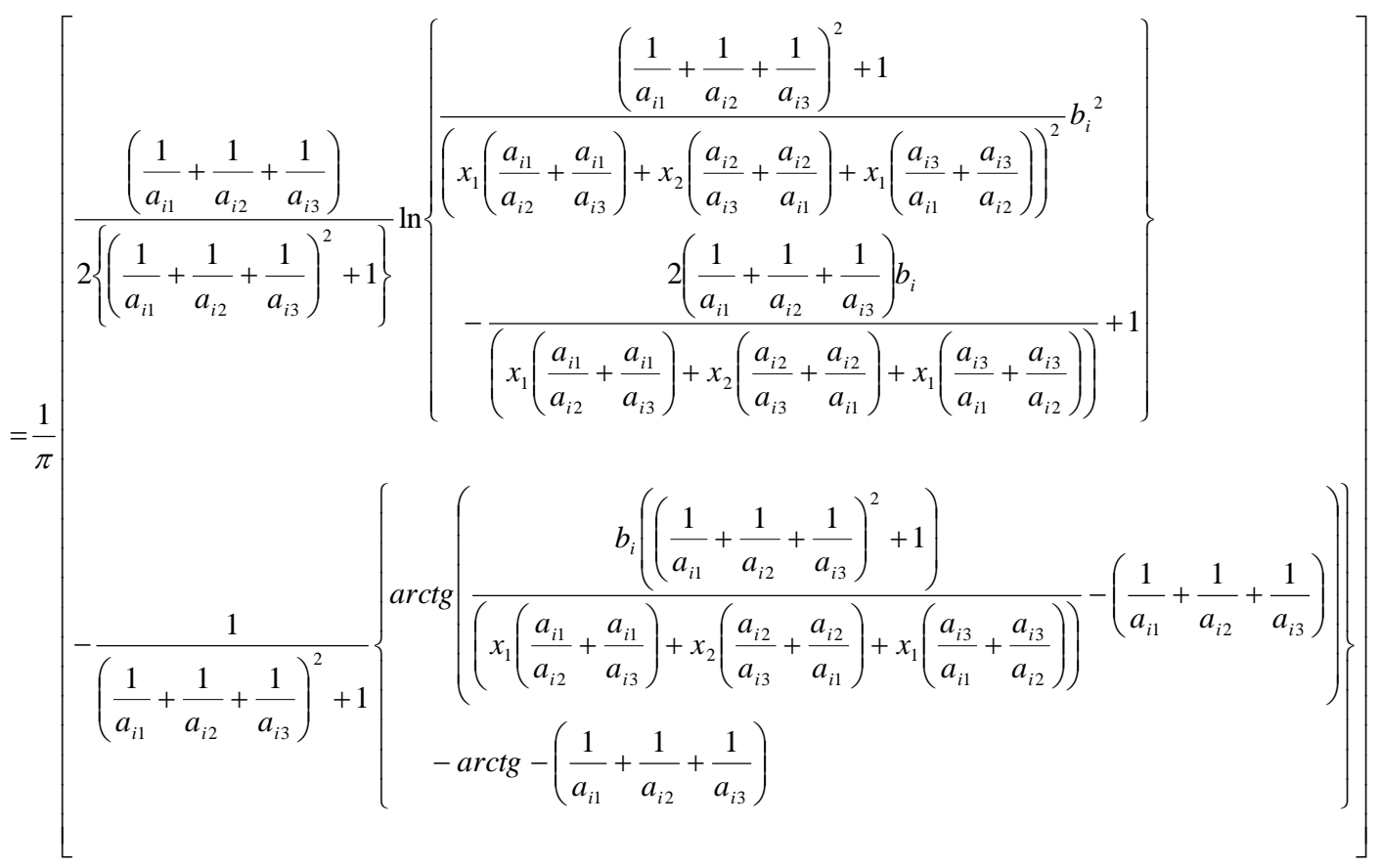

Finally we generalize the results to ' $n$ ' independent random variables and the result may be stated as follows

$$
\operatorname{Pr} o b\left(a_{i 1} x_{1}+a_{i 2} x_{2}+\ldots \ldots \ldots \ldots \ldots \ldots . .+a_{i n} x_{n}\right) \leq b_{i} \geq 1-\alpha_{i}, i=1,2, \ldots \ldots \ldots \ldots \ldots, m
$$

Where $a_{i 1}, a_{i 2}, \ldots \ldots \ldots \ldots \ldots . . . . . ., a_{i n}$ are ' $n$ ' independent Cauchy random variables

Let $\mathrm{U}_{\mathrm{i}}=\mathrm{a}_{\mathrm{i} 1} \mathrm{x}_{1}+\mathrm{a}_{\mathrm{i} 2} \mathrm{X}_{2}+\ldots \ldots \ldots \ldots+\mathrm{a}_{\mathrm{in}} \mathrm{X}_{\mathrm{n}}$

Then, $\int_{0}^{b_{i}} f\left(u_{i}\right) d u_{i}=\frac{1}{\pi} \int_{0}^{b_{i}} \frac{\left(A u_{i}-B\right)}{\left(\left(A u_{i}-B\right)^{2}+u_{i}^{2}\right)} d u_{i}$

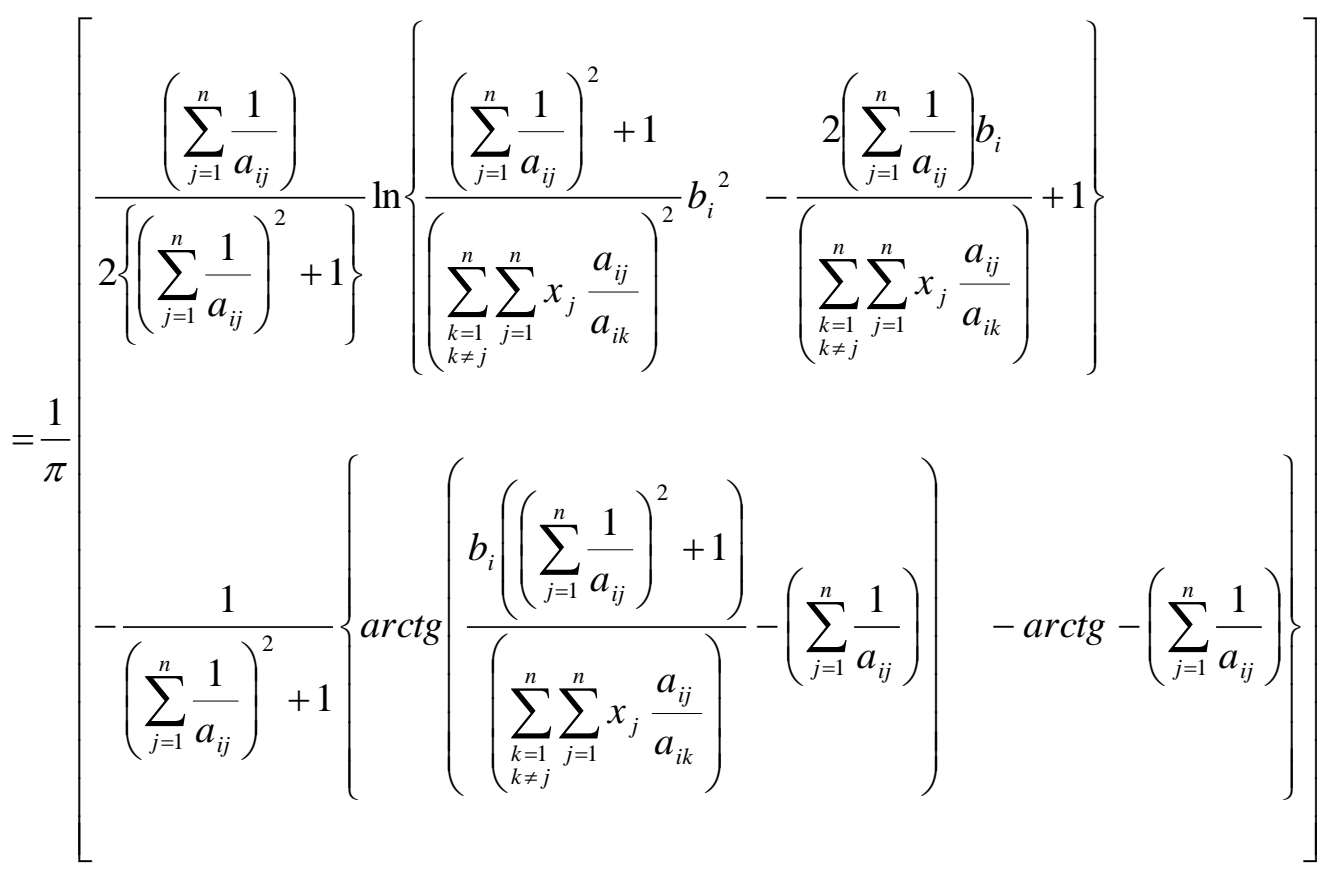




\section{Deterministic model of stochastic linear programming}

Assuming $\mathrm{a}_{\mathrm{ij}}$ to be independent Cauchy random variables, the stochastic linear programming model as stated in (1), (2) and (3) can be converted to a deterministic linear programming problem as

$$
\begin{aligned}
& \text { Maximize } \\
& \qquad E[Z]=\sum_{j=1}^{n} E\left[c_{j}\right] x_{j}
\end{aligned}
$$

Subject to the constraints,

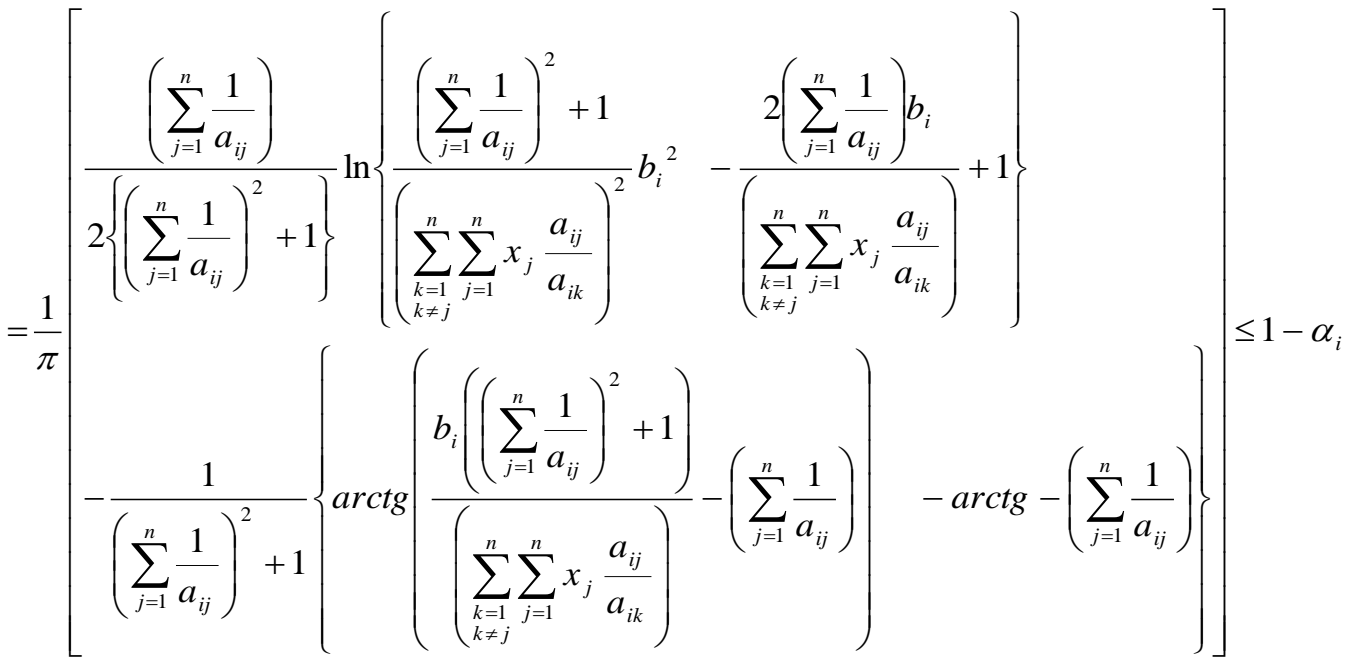

$$
\begin{aligned}
& x_{j} \geq 0 \quad, j=1,2, \ldots \ldots \ldots \ldots \ldots \ldots \ldots, n
\end{aligned}
$$

Then we present a numerical example to illustrate the methodology.

Let, $a_{11}=5, a_{12}=4, a_{13}=8, a_{21}=10, a_{22}=2, a_{23}=20$

$\mathrm{b}_{1}=10, \mathrm{~b}_{2}=20, \alpha_{1}=0.05$ and $\alpha_{2}=0.1$

Maximize, $\mathrm{Z}=5 x_{1}+6 x_{2}+3 x_{3}$

Subject to the constraints,

$$
\frac{1}{\pi}\left[\begin{array}{c}
\frac{0.575}{2.66125} \ln \left\{\frac{133.0625}{\left(1.875 x_{1}+1.3 x_{2}+3.6 x_{3}\right)^{2}}-\frac{11.5}{\left(1.875 x_{1}+1.3 x_{2}+3.6 x_{3}\right)}+1\right\} \\
-\left(\frac{1}{1.330625}\right)\left\{\operatorname{arctg} \frac{133.0625}{\left(1.875 x_{1}+1.3 x_{2}+3.6 x_{3}\right)}-(0.575)-\operatorname{arctg}(-0.575)\right\}
\end{array}\right] \leq 0.05
$$

$$
\begin{gathered}
\frac{1}{\pi}\left[\begin{array}{c}
\frac{0.65}{2.845} \ln \left\{\frac{56.9}{\left(5.5 x_{1}+0.3 x_{2}+12 x_{3}\right)^{2}}-\frac{26}{\left(5.5 x_{1}+0.3 x_{2}+12 x_{3}\right)}+1\right\} \\
-\left(\frac{1}{1.4225}\right)\left\{\operatorname{arctg} \frac{28.45}{\left(5.5 x_{1}+0.3 x_{2}+12 x_{3}\right)}-(0.65)-\operatorname{arctg}(-0.65)\right\}
\end{array}\right] \leq 0.01 \\
x_{1}, x_{2} \text { and } x_{3} \geq 0
\end{gathered}
$$


By using the Genetic Programming we found the optimum solution for the above program is

$$
x_{1}=1.741, \quad x_{2}=1.748, \quad x_{3}=1.727 \quad \text { and } \quad E[Z]=23.886
$$

\section{References}

1. Biswal, M.P, Biswal, N. P. and Duan, Li (1998). Probabilistic Linear Programming Problems with exponential random variables: A technical note. European Journal of Operational Research, Vol. 11, Page 589-597.

2. Chankong, V. and Haimes, Y.Y (1983). Multi objective Decision making theory and Methodology, North-Holland, Amsterdam.

3. Charnes, A. and Cooper, W.W (1963). Deterministic equivalents for optimizing and satisfying under chance constraints, Operations Research., Vol. 11, Page 1839.

4. Charnes, A. and Cooper, W.W (1959). Chance Constrained Programming, Management Science. Vol. 6, Page 227-243.

5. Goicoechea, A and Duckstein, L (1987). Non-normal deterministic equivalents and a transformation in stochastic programming. Applied Mathematics and Computation, Vol.21, Page 51-72.

6. Goicoechea, A., Hansen, D. R. and Duckstein, L (1982). Multi objective Decision Analysis with Engineering and Business Applications., Wiley, New York.

7. Infagen, G (1993). Planning under uncertainty: Solving Large-Scale Stochastic Linear Program. Boyd and Fraser Publishing Company, Massachusetts.

8. Kall, P and Wallace, S. W (1994). Stochastic Programming, Wiley, New York.

9. Kambo, N. S (1984). Mathematical Programming Techniques, Affiliated EastWest Press Private Limited.

10. Stancu-Minasian, I. M. and Wets, M. J (1976).A research bibliography in Stochastic Programming, 1955-1975.Operation Research, Vol.24, Page 10781119.

11. Vajda, S (1972). Probabilistic Programming, Academic Press, New York and London.

12. Verma, R., Biswal, M. P and Biswas, A (1996). Fuzzy programming approach to probabilistic multi-objective transportation problems with pareto optimum solutions. The Journal of Fuzzy Mathematics, Vol.4, Page 301-314.

13. Winston, W. L (1995). Introduction to Mathematical Programming and Algorithms, 2nd edn, Duxbury Press, Belmont, California. 